\title{
Flavonoid intake and the risk of ischaemic stroke and CVD mortality in middle-aged Finnish men: the Kuopio Ischaemic Heart Disease Risk Factor Study
}

\author{
Jaakko Mursu ${ }^{1}$, Sari Voutilainen ${ }^{1}$, Tarja Nurmi ${ }^{1}$, Tomi-Pekka Tuomainen ${ }^{1}$, Sudhir Kurl ${ }^{1}$ \\ and Jukka T. Salonen ${ }^{1,2}$ \\ ${ }^{1}$ Research Unit of Public Health, School of Public Health and Clinical Nutrition, University of Kuopio, PO Box 1627, \\ Kuopio 70211, Finland \\ ${ }^{2}$ Oy Jurilab Ltd, Microkatu 1, Kuopio 70210, Finland
}

(Received 18 April 2007 - Revised 6 December 2007 - Accepted 20 December 2007 - First published online 1 April 2008)

The role of flavonoids in CVD, especially in strokes, is unclear. Our aim was to study the role of flavonoids in CVD. We studied the association between the intakes of five subclasses (flavonols, flavones, flavanones, flavan-3-ols and anthocyanidins), a total of twenty-six flavonoids, on the risk of ischaemic stroke and CVD mortality. The study population consisted of 1950 eastern Finnish men aged 42-60 years free of prior CHD or stroke as part of the prospective population-based Kuopio Ischaemic Heart Disease Risk Factor Study. During an average follow-up time of $15 \cdot 2$ years, 102 ischaemic strokes and 153 CVD deaths occurred. In the Cox proportional hazards model adjusted for age and examination years, BMI, systolic blood pressure, hypertension medication, serum HDL- and LDL-cholesterol, serum TAG, maximal oxygen uptake, smoking, family history of CVD, diabetes, alcohol intake, energy-adjusted intake of folate, vitamin E, total fat and saturated fat intake (percentage of energy), men in the highest quartile of flavonol and flavan-3-ol intakes had a relative risk of 0.55 (95\% CI 0.31, 0.99) and 0.59 (95\% CI 0.30, 1.14) for ischaemic stroke, respectively, as compared with the lowest quartile. After multivariate adjustment, the relative risk for CVD death in the highest quartile of flavanone and flavone intakes were 0.54 (95\% CI 0.32, 0.92) and 0.65 (95\% CI 0.40, 1.05), respectively. The present results suggest that high intakes of flavonoids may be associated with decreased risk of ischaemic stroke and possibly with reduced CVD mortality.

Cardiovascular disease: Cohort: Diet: Flavonoids: Kuopio Ischaemic Heart Disease Risk Factor (KIHD) Study: Middle-aged men: Stroke

High consumption of fruit and vegetables has been associated with reduced risk of chronic diseases such as CVD ${ }^{(1)}$. Flavonoids are a large group of polyphenolic compounds $(>5000$ identified) abundant in vegetables, fruits, tea and red wine, and thus it has been suggested that they may contribute to the protective effect of these foods ${ }^{(2)}$. Several cohort studies have suggested that high intake of flavonoids may decrease the risk of $\mathrm{CHD}^{(3-16)}$. However, the role in stroke has been studied much less ${ }^{(8,11-13,17,18)}$.

Out of thousands of identified flavonoids, twenty to thirty compounds from five subclasses (flavonols, flavones, flavanones, flavan-3-ols and anthocyanidins) have been estimated to contribute significantly to daily dietary intake and thus potentially have effects on health ${ }^{(19)}$. Epidemiological studies published so far have, however, concentrated mainly on two subclasses: flavonols and flavones ${ }^{(3-13)}$.

In 2003, the US Department of Agriculture published updated food composition data which contained five subclasses: flavones, flavonols, flavan-3-ols, flavanones and anthocyanidins; a total of twenty-six flavonoids ${ }^{(20)}$. The aim of the present study was to use an updated flavonoid database to investigate the associations between the intake of flavonoids and the risk of stroke and CVD mortality in middle-aged Finnish men. Isoflavonoids were not included in the present study as their intake is very low in Finland.

\section{Material and methods}

\section{Study population}

The Kuopio Ischaemic Heart Disease Risk Factor Study (KIHD) is an ongoing population-based study designed to investigate risk factors for CVD, atherosclerosis and related outcomes in middle-aged men from eastern Finland ${ }^{(21)}$. The study was approved by the Research Ethics Committee of the University of Kuopio. All study subjects gave their written informed consent. A total of 2682 participants $(82.9 \%$ of those eligible), aged 42, 48, 54 or 60 years, were enrolled in the study between March 1984 and December 1989. Men who had history of CHD or stroke were excluded from the study and after exclusion complete data were available for 1950 subjects. 


\section{Measurements}

Blood samples were taken between 08.00 and 10.00 hours. Subjects were instructed to abstain from ingesting alcohol for $3 \mathrm{~d}$, and from smoking and eating for $12 \mathrm{~h}$. After the subject had rested in the supine position for $30 \mathrm{~min}$, blood was drawn with Terumo Venoject (Leuven, Belgium) vacuum tubes. No tourniquet was used.

The main serum lipoprotein fractions, LDL-cholesterol, HDL-cholesterol (Kone Instruments, Espoo, Finland) and TAG (Boehringer Mannheim, Mannheim, Germany) were determined from fresh serum samples using combined ultracentrifugation and precipitation. Maximal oxygen uptake was measured as previously described ${ }^{(22)}$. Diabetes was assessed by previous diagnosis of diabetes or fasting blood glucose concentration $\geq 6.7 \mathrm{mmol} / \mathrm{l}$. BMI was computed as the ratio of weight to the square of height $\left(\mathrm{kg} / \mathrm{m}^{2}\right)$. Resting systolic blood pressure was measured after supine rest of $5 \mathrm{~min}$, three measurements in supine, one on standing and two in the sitting position with 5 min intervals with a random-zero mercury sphygmomanometer (Hawksley, UK). The mean of all six measurements was used as the systolic blood pressure. The number of cigarettes, cigars and pipefuls of tobacco currently smoked daily, duration of regular smoking in years, alcohol consumption, history of myocardial infarction, angina pectoris and medication were recorded with a self-administered questionnaire, which was checked by an interviewer. Repeated interviews to obtain medical history of CHD were conducted by a physician. The family history of CHD was defined as positive if either father, mother, sister or brother of the subject had a history of CHD. A subject was defined a smoker if he had ever smoked on a regular basis and had smoked cigarettes, cigars or a pipe within the past $30 \mathrm{~d}$.

\section{Collection and classification of coronary events and strokes}

The collection of data and the diagnostic classification of strokes between 1984 and 1992 were carried out as a part of the multinational WHO MONICA (MONItoring of Trends and Determinants in CArdiovascular Diseases) project, in which detailed information of strokes were collected prospectively $^{(23)}$. In the FINMONICA study, regional coronary and stroke register teams collected data on strokes from hospitals and wards of health centres and classified the events, as explained in detail previously ${ }^{(24)}$. Data on strokes from the beginning of 1993 were obtained by computer linkage to the national hospital discharge and death registers. Strokes were collected and classified by a neurologist using the same procedures as in the FINMONICA study ${ }^{(24)}$. The diagnosis of stroke was based on a sudden onset of clinical signs of focal or global disturbance of cerebral function lasting more than $24 \mathrm{~h}$ (except in the cases of sudden death or if the development of symptoms was interrupted by surgical intervention) with no apparent cause other than vascular origin. Each suspected stroke (Ninth International Classification of Diseases (ICD-9) codes 430-438 and Tenth International Classification of Diseases (ICD-10) codes I60-I68 and G45-G46) was classified into (1) a definite stroke, (2) no stroke or (3) an unclassifiable event. Each definite stroke was classified into (1) an ischaemic stroke (ICD-9 codes 433-434, ICD-10 code I63) or (2) a haemorrhagic stroke (ICD-9 codes 430443 , ICD-10 codes $\mathrm{I} 60-\mathrm{I} 61)^{(25)}$.
CVD deaths were ascertained by computer linkage to the national death registry using the Finnish social security number. All CVD deaths that occurred from the study entry to 31 December 2004 were included. There were no losses to follow-up. CVD deaths were coded according to the ICD9 (code numbers 390-459) and the ICD-10 (code numbers I00-I99).

In the present study, definite ischaemic strokes and CVD deaths were used as outcome events. If a subject had multiple strokes during the follow-up, the first was considered as the end-point.

\section{Assessment of nutrient intake}

The consumption of foods was assessed at the time of blood sampling at the study baseline with an instructed $4 \mathrm{~d}$ food recording by household measures. The instructions were given and the completed food records were checked by a nutritionist. The intakes of nutrients were estimated using the Nutrica ${ }^{\circledR}$ version 2.5 software. Under- or over-reporters were not excluded from the analyses. The intakes of nutrients used in the Cox models were energy-adjusted by the residual method $^{(26)}$. The measurement of total, subclass and individual flavonoid intake was based on the US Department of Agriculture flavonoid database ${ }^{(20)}$. The database includes a total of twenty-six flavonoids from five subclasses: flavonols (quercetin, kaempferol, myricetin, isorhamnetin), flavones (luteolin, apigenin), flavanones (hesperetin, naringenin, eriodictyol), flavan-3-ols $((+)$-catechin, $(+)$-gallocatechin, ( -$)$-epicatechin, (-)-epigallocatechin, (-)-epicatechin-3-gallate, (-)epigallocatechin-3-gallate, theaflavin, theaflavin-3-gallate, theaflavin-3'-gallate, theaflavin-3,3'-digallate, thearubigins) and anthocyanidins (cyanidin, delphinidin, malvidin, pelargonin, peonidin, petunidin).

\section{Statistical analysis}

The distributions were expressed as means and standard deviations. The means were compared using ANOVA and categorical variables using $\chi^{2}$ tests. Subjects were classified into quartiles according to their energy-adjusted flavonoid intake and the relationship of flavonoid intake with the risk of CVD was analysed using Cox proportional hazards models. Model 1 was adjusted for age and examination years, and model 2 in addition BMI, systolic blood pressure, hypertension medication, serum HDL- and LDL-cholesterol, serum TAG, maximal oxygen uptake, smoking, family history of CVD, diabetes, alcohol intake, energy-adjusted intake of folate, vitamin E, total fat and saturated fat intake (percentage of energy). Relative risks (RR), adjusted for other risk factors, were estimated as antilogarithms of coefficients for independent variables. The CI were estimated based on the assumption of asymptotic normality of estimates. All statistical tests were two-tailed. Data were analysed using SPSS for Windows version 11.5 statistical software (SPSS Inc., Chicago, IL, USA).

\section{Results}

The mean flavonoid intake was $139.3 \mathrm{mg} / \mathrm{d}$, and out of the total intake, the flavan-3-ol subclass contributed $85.9 \%$ $(119.7 \mathrm{mg} / \mathrm{d})$, flavonols $7.2 \%(10.0 \mathrm{mg} / \mathrm{d})$, anthocyanidins 
$4.5 \%(6.2 \mathrm{mg} / \mathrm{d})$, flavonones $2.2 \%(3.1 \mathrm{mg} / \mathrm{d})$ and flavones $0.2 \%(0.3 \mathrm{mg} / \mathrm{d})$. The mean age of the study population was 52.4 (SD 5.3) years and during the average follow-up time of $15 \cdot 2$ years, men with no previous CHD or stroke experienced 102 ischaemic strokes and 153 CVD deaths. When comparing in quartiles of flavonoid intake, the men with the highest quartile of intake were more likely to be non-smokers, tended to have lowest alcohol, total fat and SFA intakes, and higher intakes of folate, fibre, vitamins $\mathrm{C}$ and $\mathrm{E}$, when compared with the lower quartiles (Table 1).

In a Cox proportional hazards model adjusted for age and examination years, the RR for the intakes of flavonol and flavan-3-ols subclasses were $0.52(95 \%$ CI $0.30,0.90)$ in the highest quartile of flavonol intake $v$. the lowest, and for flavan-3-ols 0.52 (95\% CI 0.28, 0.99), respectively (Table 2). After multivariate adjustment, the RR for the ischaemic stroke in the highest quartile of flavonol and flavan-3-ol intakes were 0.55 (95\% CI $0.31,0.99)$ and 0.59 (95\% CI $0.30,1.14$ ), respectively. The total intake or the intakes of other subclasses, anthocyanidins, flavanones or flavones, were not associated with the risk of ischaemic stroke.

After adjustment for age and examination years, total or flavonoid subclasses were not associated with CVD mortality. After multivariate adjustment, the RR for CVD death in the highest quartile of flavanone intake was 0.54 (95\% CI 0.32 ,
0.92) when compared with the lowest quartile. A non-significant trend was also found for flavones, the RR was 0.65 (95\% CI 0.40, 1.05).

\section{Discussion}

The aim of the present study was to investigate the role of the most commonly consumed flavonoids and the risk of ischaemic stroke and CVD mortality in a population-based cohort of middle-aged Finnish men. The main finding was that out of five studied flavonoid subclasses, the high intakes of flavonols, and less strongly flavan-3-ols, were associated with decreased risk of ischaemic stroke. The intakes of other flavonoid subclasses, anthocyanidins, flavanones or flavones, were not associated with the risk of ischaemic stroke. The intakes of flavonones and flavones may be associated with decreased risk of CVD mortality. However, the inverse association found for flavanones was not consistent, while for flavones the association was consistent, but not significant. In addition, the intakes of these subclasses were relatively low, $2 \cdot 2 \%$ for flavanones and $0.2 \%$ for flavones, and therefore it is difficult to verify whether the associations found are true or the result of chance, for example. Further studies are also needed to evaluate whether the possible protective effect is related to some specific type of CVD.

Table 1. Characteristics of the 1950 study subjects according to the energy-adjusted flavonoid intake*

(Mean values and standard deviations)

\begin{tabular}{|c|c|c|c|c|c|c|c|c|c|}
\hline & \multicolumn{8}{|c|}{ Quartiles of flavonoid intake $(\mathrm{mg} / \mathrm{d})$} & \multirow[b]{3}{*}{$P \dagger$} \\
\hline & \multicolumn{2}{|c|}{1 (lowest) } & \multicolumn{2}{|c|}{2} & \multicolumn{2}{|c|}{3} & \multicolumn{2}{|c|}{4 (highest) } & \\
\hline & Mean & SD & Mean & SD & Mean & SD & Mean & SD & \\
\hline Mean flavonoid intake, not energy-adjusted (mg/d) & 9.5 & $10 \cdot 8$ & 18.7 & $16 \cdot 6$ & 94.5 & 47.9 & $435 \cdot 0$ & $272 \cdot 3$ & $<0.001$ \\
\hline Mean flavonoid intake, energy-adjusted (mg/d) & $-7 \cdot 0$ & $17 \cdot 3$ & 27.4 & $9 \cdot 0$ & $99 \cdot 1$ & $40 \cdot 0$ & 433.0 & 267.7 & $<0.001$ \\
\hline Age (years) & 51.9 & $5 \cdot 4$ & $53 \cdot 2$ & $5 \cdot 3$ & 52.4 & $5 \cdot 3$ & $52 \cdot 2$ & $5 \cdot 3$ & 0.001 \\
\hline BMI $\left(\mathrm{kg} / \mathrm{m}^{2}\right)$ & $26 \cdot 7$ & 3.4 & $26 \cdot 8$ & 3.5 & $26 \cdot 7$ & 3.5 & $26 \cdot 5$ & 3.5 & 0.506 \\
\hline Waist:hip ratio & 0.95 & 0.05 & 0.95 & 0.05 & 0.95 & 0.06 & 0.94 & 0.05 & 0.493 \\
\hline Systolic blood pressure $(\mathrm{mmHg})$ & 134 & 16 & 136 & 17 & 134 & 16 & 134 & 16 & $0 \cdot 181$ \\
\hline Hypertension medication (\%) & $10 \cdot 6$ & & $14 \cdot 8$ & & $17 \cdot 4$ & & $12 \cdot 5$ & & $0 \cdot 14$ \\
\hline Serum total cholesterol $(\mathrm{mmol} / \mathrm{l})$ & $5 \cdot 86$ & 0.99 & 5.98 & $1 \cdot 13$ & $5 \cdot 80$ & 1.06 & $5 \cdot 80$ & 0.98 & 0.020 \\
\hline Serum LDL-cholesterol (mmol//) & 4.01 & 0.93 & 4.09 & 1.05 & 3.95 & 1.02 & 3.98 & 0.90 & 0.129 \\
\hline Serum HDL-cholesterol (mmol/l) & 1.33 & 0.30 & 1.30 & 0.29 & 1.30 & 0.29 & 1.29 & 0.29 & 0.327 \\
\hline Serum TAG (mmol/l) & 1.22 & 0.67 & 1.29 & 0.70 & 1.27 & 0.74 & 1.23 & 0.81 & 0.385 \\
\hline Maximal $\mathrm{O}_{2}$ uptake $(\mathrm{ml} / \mathrm{kg}$ per $\mathrm{min})$ & $32 \cdot 2$ & $7 \cdot 0$ & 31.1 & $7 \cdot 0$ & $32 \cdot 3$ & $7 \cdot 3$ & $32 \cdot 3$ & $7 \cdot 4$ & 0.028 \\
\hline Leisure-time physical activity (kJ/d) & $482 \cdot 8$ & 636.8 & 558.6 & 638.9 & $670 \cdot 7$ & 825.5 & $605 \cdot 4$ & 677.4 & $<0.001$ \\
\hline Leisure-time physical activity (kcal/d) & $115 \cdot 4$ & $152 \cdot 2$ & 133.5 & $152 \cdot 7$ & $160 \cdot 3$ & $197 \cdot 3$ & 144.7 & 161.9 & $<0.001$ \\
\hline Smokers (\%) & 38.2 & & $32 \cdot 4$ & & 28.9 & & $19 \cdot 9$ & & $<0.001$ \\
\hline Family history of IHD (\%) & $44 \cdot 1$ & & $46 \cdot 6$ & & $49 \cdot 2$ & & $44 \cdot 8$ & & 0.382 \\
\hline Diabetes (\%) & 3.9 & & 4.7 & & $3 \cdot 1$ & & 4.9 & & 0.444 \\
\hline Alcohol intake ( $\mathrm{g} /$ week) & $87 \cdot 1$ & $136 \cdot 1$ & $72 \cdot 3$ & $106 \cdot 0$ & $69 \cdot 7$ & $115 \cdot 7$ & $60 \cdot 5$ & $95 \cdot 2$ & 0.003 \\
\hline Total fat intake (\% of total energy) & $40 \cdot 2$ & $6 \cdot 4$ & 38.6 & $6 \cdot 4$ & 37.5 & $6 \cdot 1$ & $38 \cdot 1$ & $5 \cdot 6$ & $<0.001$ \\
\hline SFA intake ( $\%$ of total energy) & $19 \cdot 2$ & 4.3 & 17.7 & $4 \cdot 1$ & $17 \cdot 1$ & $4 \cdot 1$ & $17 \cdot 5$ & 3.8 & $<0.001$ \\
\hline Fibre intake $(\mathrm{g} / \mathrm{d})$ & $23 \cdot 6$ & $7 \cdot 7$ & 24.8 & $6 \cdot 3$ & $26 \cdot 2$ & $7 \cdot 2$ & $26 \cdot 1$ & $7 \cdot 1$ & $<0.001$ \\
\hline Folate intake $(\mu \mathrm{g} / \mathrm{d})$ & 236 & 54 & 251 & 52 & 264 & 60 & 274 & 61 & $<0.001$ \\
\hline Vitamin C intake $(\mathrm{mg} / \mathrm{d})$ & $56 \cdot 5$ & $36 \cdot 8$ & $73 \cdot 2$ & 47.5 & 88.2 & $59 \cdot 2$ & $77 \cdot 7$ & $53 \cdot 3$ & $<0.001$ \\
\hline Vitamin E intake (mg/d) & 8.4 & $2 \cdot 6$ & 8.9 & $2 \cdot 2$ & $9 \cdot 4$ & $2 \cdot 4$ & $9 \cdot 3$ & 2.5 & $<0.001$ \\
\hline \multicolumn{10}{|l|}{ Events } \\
\hline No. of CVD deaths & 33 & & 57 & & 32 & & 31 & & 0.003 \\
\hline$\%$ CVD deaths & $6 \cdot 7$ & & 11.8 & & $6 \cdot 6$ & & 6.4 & & 0.003 \\
\hline No. of ischaemic strokes & 24 & & 39 & & 23 & & 16 & & \\
\hline$\%$ Ischaemic strokes & 4.9 & & $8 \cdot 0$ & & $4 \cdot 7$ & & $3 \cdot 3$ & & 0.008 \\
\hline
\end{tabular}

${ }^{\star}$ For details of procedures, see Material and methods.

$\dagger P$ value from the ANOVA for continuous variables or from $\chi^{2}$ test for discrete variables. 
Table 2. Multivariate-adjusted relative risks (RR) of CVD according to the quartiles of energy-adjusted flavonoid intake*

(Relative risk values and $95 \% \mathrm{Cl}$ )

\begin{tabular}{|c|c|c|c|c|c|c|c|c|}
\hline & \multicolumn{7}{|c|}{ Quartiles of flavonoid intake $(\mathrm{mg} / \mathrm{d})$} & \multirow[b]{3}{*}{$P$ for trend } \\
\hline & \multirow[b]{2}{*}{1 (lowest) } & \multicolumn{2}{|r|}{2} & \multicolumn{2}{|r|}{3} & \multicolumn{2}{|c|}{4 (highest) } & \\
\hline & & $\mathrm{RR}$ & $95 \% \mathrm{Cl}$ & $\mathrm{RR}$ & $95 \% \mathrm{Cl}$ & $\mathrm{RR}$ & $95 \% \mathrm{Cl}$ & \\
\hline \multicolumn{9}{|l|}{ Ischaemic stroke } \\
\hline Flavonols & 1 & 0.68 & $0.40,1 \cdot 14$ & 0.54 & $0.30,0.95$ & 0.55 & $0.31,0.99$ & 0.027 \\
\hline Flavones & 1 & $1 \cdot 12$ & $0.60,2 \cdot 11$ & 2.05 & $1.15,3.65$ & 1.30 & $0.69,2.47$ & $0 \cdot 181$ \\
\hline Flavanones & 1 & 0.83 & $0.47,1.47$ & 0.97 & $0.56,1.71$ & 0.89 & $0.49,1.63$ & 0.870 \\
\hline Flavan-3-ols & 1 & 1.24 & $0.73,2 \cdot 10$ & 1.02 & $0.58,1.80$ & 0.59 & $0.30,1.14$ & 0.102 \\
\hline Anthocyanidins & 1 & 0.89 & $0.48,1.63$ & 1.58 & $0.91,2.71$ & 0.88 & $0.47,1.62$ & 0.813 \\
\hline Total sum of flavonoids & 1 & 1.65 & $0.98,2.79$ & 1.00 & $0.55,1.81$ & 0.71 & $0.37,1.37$ & 0.137 \\
\hline \multicolumn{9}{|l|}{ CVD mortality } \\
\hline Flavonols & 1 & 1.22 & $0.77,1.94$ & 1.42 & $0 \cdot 88,2 \cdot 28$ & 1.26 & $0.75,2 \cdot 14$ & 0.622 \\
\hline Flavones & 1 & 0.64 & $0.40,1.05$ & 0.64 & $0.40,1.02$ & 0.65 & $0.40,1.05$ & 0.333 \\
\hline Flavanones & 1 & 0.60 & $0.37,0.98$ & 0.97 & $0.62,1.50$ & 0.54 & $0.32,0.92$ & 0.266 \\
\hline Flavan-3-ols & 1 & 1.29 & $0.82,2.04$ & 1.03 & $0.64,1.65$ & 1.06 & $0.64,1.65$ & 0.694 \\
\hline Anthocyanidins & 1 & 0.51 & $0.30,0.87$ & 1.17 & $0.74,1.86$ & 0.99 & $0.62,1.85$ & 0.193 \\
\hline Total sum of flavonoids & 1 & 1.85 & $1.18,2.90$ & 1.05 & $0.63,1.74$ & 1.25 & $0.74,2.11$ & 0.730 \\
\hline
\end{tabular}

*Adjusted for age, examination years, BMI, systolic blood pressure, hypertension medication, serum HDL- and LDL-cholesterol, serum TAG, maximal oxygen uptake, smoking, CVD in family, diabetes, alcohol intake, energy-adjusted intake of folate and vitamin $\mathrm{E}$, total fat (percentage of energy) and saturated fat intake (percentage of energy). For details of procedures, see Material and methods.

The most relevant new information shown by the present study was that with updated US Department of Agriculture databases we could assess the role of five subclasses, a total of twenty-six flavonoids, instead of one or two subclasses included in most of the previous studies. Most of the previous cohorts have included only flavonols and flavones, and so far only a few have evaluated the role of flavanones ${ }^{(12,16)}$, flavan3 -ols ${ }^{(10,11,16)}$ or anthocyanidins ${ }^{(16)}$ in CVD. A recent study assessed the role of a total of seven subclasses and found flavanones, anthocyanidins and flavonoid-rich food to be associated with total, CHD and CVD mortality ${ }^{(16)}$.

The present findings, suggesting that intake of flavonoids could be associated with the risk of ischaemic stroke, but not that strongly with the risk of CVD mortality, were surprising. The aetiology of ischaemic stroke and other CVD is considered to be mainly similar and thus flavonoids were expected to be associated with CVD mortality also. One possible explanation could be that atherosclerosis might lead to stroke earlier because of the smaller diameter of the cerebral arterioles.

Seasonal variation is one of the main limitations of the $4 \mathrm{~d}$ food recording method and could have an effect on the results. In Finland the intake of fruits and vegetables is highest during the summer and lowest in the winter, and thus the date of the food recording may have biased the flavonoid intake and misclassification of the subjects. This may have attenuated the association between flavonoid intake and the risk of CVD.

The inverse association found for ischaemic stroke was limited to flavonols and less strongly to flavan-3-ols, while the intakes of the other three subclasses were not found to be associated. The lack of association could simply be due to the fact that the intake of these subclasses was very low, $10 \%$ of the total intake, which reduces the statistical power to detect a possible association. On the other hand, individual compounds or subclasses have distinct chemical properties and it is possible that the effects on human health also would differ ${ }^{(2)}$.
The present results suggesting that flavonoids may decrease the risk of ischaemic stroke, but not that strongly with CVD mortality, differ somewhat from previous findings. Cohort studies have suggested that high intake of flavonoids, and especially flavonols, may decrease the risk of CHD, but probably not the risk of stroke. Half of the published cohort studies have found high intake of flavonoids to be associated with decreased risk of $\mathrm{CVD}^{(3-14,16)}$. In a meta-analysis based on the data from seven cohorts, the high intake of flavonols was associated with $20 \%$ reduced CHD mortality ${ }^{(15)}$.

Only six cohort studies have, however, evaluated the role of flavonoids in stroke. Two of these studies found the intake of flavonols to be inversely associated with the risk of stroke ${ }^{(12,17)}$, while four studies did not find any association $^{(8,10,13,18)}$. Four of these studies assessed the role of flavonols and flavones ${ }^{(8,13,17,18)}$, one in addition flavanones ${ }^{(12)}$ and one concentrated on catechins ${ }^{(10)}$. In general, the inconsistency in the association between flavonoid intake and stroke may be related to differences in the study population (e.g. age, gender), types of strokes studied or the study setting.

The possible mechanisms by which flavonoids decrease the risk of CVD have most often been suggested to be related, for example, to their vasodilatory or antioxidant properties. Supplementation of flavonoids has been found to improve endothelial function by increasing nitric oxide production in $\operatorname{man}^{(27,28)}$. Flavonoids are strong antioxidants in vitro and in animal studies flavonoids have been found to inhibit oxidative stress as well as the progression of atherosclerosis ${ }^{(29,30)}$. The studies in man have, however, yielded conflicting results ${ }^{(31)}$. Flavonoids have been suggested also to have beneficial effects on blood pressure, platelet function and inflammation.

In the present study the intake of flavonoids was associated with lifestyle; men with higher intakes were more likely to be non-smokers and had lower intakes of alcohol, fat and SFA as well as higher intakes of folate, fibre, vitamin $\mathrm{C}$ and $\mathrm{E}$ when compared with the men with lower intakes. Therefore, we cannot exclude the possibility that despite extensive 
adjustment for CVD risk factors, the high intake of flavonoids could be an indicator of healthy lifestyle rather than a protective factor.

Mean flavonoid intake $(140 \mathrm{mg})$ in the present study was much higher than reported previously for the Finnish population (about $10-20 \mathrm{mg})^{(7,9,12)}$ and this was because the previous calculations have included mainly flavonols and flavones, while the present study also included flavan-3-ols, flavanones and anthocyanidins. In these studies the main sources of flavonols and flavones in the Finnish population have been tea, apples and onions, and citrus fruits for flavanones. One of the limitations of the present study was that we could not study the main sources of flavonoids. However, it is most likely that the main source of flavonols, flavones and flavanones have been the same, while the main source of flavan-3-ols has been tea, and berries for anthocyanidins.

Further studies are still needed to assess the role of flavonoids in CVD, especially in strokes. In addition to flavonoids, other polyphenols such as phenolic acids may also play a role in human health. Phenolic acids are rich in, for example, coffee and cereals, and these compounds contribute significantly to the daily intake of polyphenols. Therefore the databases should be updated concerning other polyphenolic compounds.

In conclusion, the present study suggests that a high intake of flavonoids decreases the risk of ischaemic stroke and possibly CVD mortality. More studies with updated flavonoid databases are needed to evaluate the role of flavonoids in CVD and especially in strokes.

\section{Acknowledgements}

This study was supported by grants from the Juho Vainio Foundation and Finnish Cultural Foundation, North-Savo Foundation (J. M.). J. T. S. was responsible for the study concept and design. S. V., T. N., T.-P. T., S. K. and J. T. S. collected the data, obtained the funding, and provided administrative, technical or material support. J. M., T. N. and S. V. analysed and interpreted the data, drafted the manuscript and provided statistical expertise. J. M., S. V., T. N., T.-P. T., S. K. and J. T. S. critically revised the manuscript for important intellectual content. None of the authors had a conflict of interests.

\section{References}

1. Ness AR \& Powles JW (1997) Fruit and vegetables, and cardiovascular disease: a review. Int J Epidemiol 26, 1-13.

2. Bravo L (1998) Polyphenols: chemistry, dietary sources, metabolism, and nutritional significance. Nutr Rev 56, 317-333.

3. Geleijnse JM, Launer LJ, Van der Kuip DA, Hofman A \& Witteman JC (2002) Inverse association of tea and flavonoid intakes with incident myocardial infarction: the Rotterdam Study. Am J Clin Nutr 75, 880-886.

4. Hertog M, Sweetnam P, Fehily AM, Elwood PC \& Kromhout D (1997) Antioxidant flavonols and ischemic heart disease in Welsh population of men: the Caerphilly study. Am J Clin Nutr 65, 1489-1494.

5. Hertog MG, Feskens EJ \& Kromhout D (1997) Antioxidant flavonols and coronary heart disease risk. Lancet 349, 699.
6. Hertog MGL, Feskens EJM, Hollman PCH, Katan MB \& Kromhout D (1993) Dietary antioxidant flavonoids and risk of coronary heart disease: the Zutphen elderly study. Lancet 342, 1007-1011.

7. Knekt P, Järvinen R, Reunanen A \& Maatela J (1996) Flavonoid intake and coronary mortality in Finland: a cohort study. $\mathrm{Br}$ Med J 312, 478-481.

8. Youchum L, Kushi LH, Meyer K \& Folsom AR (1999) Dietary flavonoid intake and risk of cardiovascular disease in postmenopausal women. Am J Epidemiol 149, 943-949.

9. Hirvonen T, Pietinen P, Virtanen MM-LO, Häkkinen S, Albanes D \& Virtamo J (2001) Intake of flavonols and flavones and risk of coronary heart disease in male smokers. Epidemiology 12, $62-67$.

10. Arts IC, Jacobs DRJ, Harnack LJ, Gross M \& Folsom AR (2001) Dietary catechins in relation to coronary heart disease death among postmenopausal women. Epidemiology 12, 668-675.

11. Arts IC, Hollman PC, Feskens EJ, Bueno de Mesquita HB \& Kromhout D (2001) Catechin intake might explain the inverse relation between tea consumption and ischemic heart disease: the Zutphen Elderly Study. Am J Clin Nutr 74, 227-232.

12. Knekt P, Kumpulainen J, Jarvinen R, Rissanen H, Heliovaara M, Reunanen A, Hakulinen T \& Aromaa A (2002) Flavonoid intake and risk of chronic diseases. Am J Clin Nutr 76, $560-568$.

13. Sesso HD, Gaziano JM, Liu S \& Buring JE (2003) Flavonoid intake and the risk of cardiovascular disease in women. $\mathrm{Am} \mathrm{J}$ Clin Nutr 77, 1400-1408.

14. Rimm E, Katan M, Ascherio A, Stampfer MJ \& Willet WC (1996) Relation between intake of flavonoids and risk of coronary heart disease in male health professionals. Ann Intern Med 125, 384-389.

15. Huxley RR \& Neil HA (2003) The relation between dietary flavonol intake and coronary heart disease mortality: a metaanalysis of prospective cohort studies. Eur J Clin Nutr 57, 904-908.

16. Mink PJ, Scrafford CG, Barraj LM, Harnack L, Hong CP, Nettleton JA \& Jacobs DRJ (2007) Flavonoid intake and cardiovascular disease mortality: a prospective study in postmenopausal women. Am J Clin Nutr 85, 895-909.

17. Keli SO, Hertog MGL, Feskens EJM \& Kromhout D (1996) Dietary flavonoids, antioxidant vitamins and incidence of stroke. Arch Intern Med 154, 637-642.

18. Hirvonen T, Virtamo J, Korhonen P, Albanes D \& Pietinen $\mathrm{P}$ (2000) Intake of flavonoids, carotenoids, vitamins C and E, and risk of stroke in male smokers. Stroke 31, 2301-2306.

19. Dwyer J \& Peterson JJ (2002) Measuring flavonoid intake: need for advanced tools. Public Health Nutr 5, 925-930.

20. US Department of Agriculture (2003) USDA database for the flavonoid content of selected foods. http://www.nal.usda.gov (accessed 12 August 2006).

21. Salonen JT (1988) Is there continuing need for longitudinal epidemiological research? The Kuopio Ischaemic Heart Disease Risk Factor Study. Ann Clin Res 20, 46-50.

22. Lakka TA, Laukkanen JA, Rauramaa R, Salonen R, Lakka H-M, Kaplan GA \& Salonen JT (2001) Cardiorespiratory fitness and the progression of carotid atherosclerosis in middle-aged men. Ann Intern Med 134, 12-20.

23. Tunstall-Pedoe H, Kuulasmaa K, Amouyel p, Arveiler D, Rajakangas AM \& Pajak A (1994) Myocardial infarction and coronary deaths in the World Health Organization MONICA Project. Registration procedures, event rates, and case-fatality rates in 38 populations from 21 countries in four continents. Circulation 90, 583-612.

24. Tuomilehto J, Rastenyte D, Sivenius J, et al. (1996) Ten-year trends in stroke incidence and mortality in the FINMONICA Stroke Study. Stroke 27, 825-832. 
25. Kurl S, Laukkanen JA, Niskanen L, Rauramaa R, Tuomainen TP, Sivenius J \& Salonen JT (2005) Cardiac power during exercise and the risk of stroke in men. Stroke 36, 820-824.

26. Willet W \& Stampfer M (1998) Implications of total energy intake for epidemiologic analyses. In Nutritional Epidemiology, pp. 273-301 [W Willet, editor]. New York: Oxford University Press.

27. Hodgson JM, Burke V \& Puddey IB (2005) Acute effects of tea on fasting and postprandial vascular function and blood pressure in humans. J Hypertens 23, 47-54.

28. Vlachopoulos C, Aznaouridis K, Alexopoulos N, Economou E Andreadou I \& Stefanadis C (2005) Effect of dark chocolate on arterial function in healthy individuals. Am J Hypertens $\mathbf{1 8}$ $785-791$.
29. Hayek T, Furhman B, Vaya J, Rosenblat M, Belinky P, Coleman R, Elis A \& Aviram M (1997) Reduced progression of atherosclerosis in apolipoprotein E-deficient mice following consumption of red wine, or its polyphenols quercetin or catechin, is associated with reduced susceptibility of LDL to oxidation aggregation. Arterioscler Thromb Vasc Biol 17, 2744-2752.

30. Fuhrman B, Volkova N, Coleman R \& Aviram M (2005) Grape powder polyphenols attenuate atherosclerosis development in apolipoprotein $\mathrm{E}$ deficient (E0) mice and reduce macrophage atherogenicity. $J$ Nutr 135, 722-728.

31. Williamson G \& Manach C (2005) Bioavailability and bioefficacy of polyphenols in humans. II. Review of 93 intervention studies. Am J Clin Nutr 81, 243-255. 